International Journal of Engineering \& Technology, $10(2)(2021) 204-207$
SPC
International Journal of Engineering \& Technology
Website: www.sciencepubco.com/index.php/IJET
Research paper

\title{
Safety climate analysis at two thermal power plants
}

\author{
Basil Marhoon ${ }^{1}$, Ahmed Y. Abdalla ${ }^{1 *}$ \\ ${ }^{1}$ Mechanical Department, College of Engineering, University of Bahrain, Kingdom of Bahrain \\ *Corresponding author E-mail: ayabdalla@uob.edu.bh
}

\begin{abstract}
The present work evaluates the safety climate as it reflects safety culture and is considered as a fast tool for assessing safety culture with minimum resources required. The evaluation is done for a private thermal combined cycle power company, Plant A. Another private company is also producing electric energy and water desalination, Plant B. Both plants are in Bahrain and under the management of one company. The evaluation is done through questionnaires, including all the staff of 210 employees. The questionnaire used in the present work is based on Nordic Safety Climate Questionnaire (NOSACQ-50) and modified by the help of the health and safety department of Plant A to be suitable to the power plant. Also, interviews are conducted to validate and support the questionnaire. Analysis has been done by different statistical methods using Microsoft Excel. The overall safety climate level of the two plants was found to be above average. The overall mean score of all results is 3.67 on a scale of 1 to 5 . The mean score for most of the dimensions was higher than 3 , which is considered a positive result. The level of safety for the daytime workers was found to be greater than the safety level of the shift workers. Also, the study reveals some factors that affect the safety climate level, such as, the nature of the work, the experience and the number of safety workshops attended.
\end{abstract}

Keywords: Safety Culture; Safety Climate; Safety Climate Level; Safety Climate Questionnaire.

\section{Introduction}

Human activities are in four sectors: domestic, agricultural, commercial and industrial. The need to be safe while engaging in any activity is a part of human nature. Therefore, the issue of safety is a constant human concern. In the industrial sector, where significant hazards occur, the need for safety requires utmost attention. It is essential for humans to feel safe while doing any activity. It is important to have a safety system in any working place, with reflective indicators that can be measured and monitored to improve it.

Nowadays, there is a movement away from safety measures based on retrospective data called 'lagging indicators' such as fatalities and time lost in accidents towards what is called 'leading indicators' such as safety audits or measurements of the safety climate. The leading indicators can enhance the process of finding weaknesses without risking the failure of the system (Flin et al., 2000).

So studying the safety culture can be a strong method to detect the weaknesses in the safety system to prevent any occurrence of unsafe events in the future. Safety culture and safety climate are concepts that today attract much attention across a broad number of industries and sectors (Kines et al., 2011). Identifying the level of safety culture is difficult and takes a long time, so evaluating the safety climate is more common, which is considered as a reflection or snapshot of the safety culture, so safety climate is considered as an alternative safety indicator (Guldenmund, 2010; Schein, 2010)

Generally, the improvement of the safety culture includes a) determining the present level of safety climate, b) selecting the wanted level of safety climate, c) creating an evaluation action plan to improve the safety climate level.

The present work uses safety climate questionnaire and interviews in the power generation and water production industries in Bahrain. It involves two plants: plant A for power generation and plant B for power generation and water production. Both plants work in a combined cycle and belong to the same mother company. Plant A produces $950 \mathrm{MW}$ of electricity, while Plant B produces 1227 MW of electricity and 48 million gallon/day (MGD) of distillate water. The desalination technique is reverse osmosis (RO). Both plants generate electric power from two blocks. Each block has two gas turbines and one steam turbine. The gas turbines can run in simple mode or combined cycle mode to supply the required heat to generate the steam required to run the steam turbine. All gas turbines are fired by natural gas as primary fuel or diesel as emergency fuel. The present work aims to evaluate the safety climate level, figure out the factors affecting the safety level, detect sub-climate existence between departments, and find out the weaknesses in the safety system in these two plants.

\section{Materials and methods}

The general steps of this work start with performing a literature review to get an idea about the targeted company, safety culture, safety climate and the methods to evaluate safety climate. Next, a meeting was arranged with the general manager and head of health, safety, and environment (HSE) department. Then, the measuring tool design process was done through multiple stages. The final step was designing 
and conducting a questionnaire to evaluate the safety climate level and performing interviews to get a better understanding of the questionnaire results.

\subsection{Questionnaire}

The questionnaire is a good measuring tool to evaluate the safety climate (Cooper, 2000; CANSO, 2008). So, prior to starting the evaluation, a meeting was arranged with representatives from the company's safety department and managers of other departments in the two power plants. In the meeting, a comparison between different questionnaires was carried out. Then, one questionnaire was chosen, namely NOSACQ-50 (Kines et al., 2011), as the main structure to formulate a questionnaire that fit the power plants: plants A and B. Then many modifications were done by comparison with other questionnaires and by seeking feedback from the Health, Safety and Environment (HSE) department.

The validation process is done through two steps. The first step seeks feedback from the HSE department as experts in the field. The second step performs a pilot study to improve the questionnaire.

A pilot study was conducted to identify any mysterious questions, spot the weaknesses, ensure easy wording, and estimate the time required for questionnaire completion. The pilot study included 20 participants. In 2008 Connelly specifies that $10 \%$ of the total population is ideal to use for the pilot study. Here, the pilot study was repeated two times. Every time, notes were taken to modify the questionnaire, then the final version was achieved. The average time required to fill in the questionnaire was found to be 10.6 minutes.

In the current work, to check the questionnaire stability, a test-retest was done. The number of participants taken is equal to the number of those used in the pilot test and chosen randomly. For the time between the two trials, there was no evidence of the correct time (Parsian and Dunning, 2009), so it is better to take a short time. In the present work, the time has been set to ten days. The Interclass Correlation Coefficient (ICC) was calculated to determine the stability of the questionnaire, which reflects its reliability. In addition to the stability test, the Cronbach alpha coefficient was also calculated to measure the internal consistency, which again reflects the reliability of the questionnaire.

The designed questionnaire for the present work consists of seven dimensions and fifty items plus background questions used to determine the factors that affect the safety climate level. All items are five points on the Likert scale, and it consists of positive and negative questions. The positive questions scored from 1 to 5 , with 1 for strongly disagree and 5 for strongly agree. The negative questions scored inversely from 5 to 1 , with 5 for strongly disagree and 1 for strongly agree. A mean score over 3 is considered a positive result since it is the mean value between the highest and lowest scores (Bergh, 2011; Kinik 2010; Kines et al., 2011).

The seven dimensions are classified as: Management safety priority and ability (9 items), Management safety empowerment (7 items), Management safety judgment (6 items), Workers' safety commitment (6 items), Workers' safety priority and risk non-acceptance (7 items), Peer safety communication learning and trust in safety ability (8 items), and Workers' trust in the efficiency of safety systems (7 items). The sample size is 210 employees, which is all the population to increase the accuracy of the results.

\subsection{Interviews}

The second tool to evaluate the safety climate is interview (Bergh, 2011). The questions used in the interview were chosen with the help of the HSE department of the company. The interview questions were based on the questions of the designed questionnaire and were used to seek more explanation to some items in the questionnaire. The interviews are structured, involved one person at a time, and consist of open-ended questions. It is structured to limit the time required. Also, it involves one person at a time to increase the level of confidentiality and let the interviewee talk freely. Finally, it consists of open-ended questions to seek the opinions of the interviewee. The sample size was 30 employees, and they were chosen by the stratified sampling method to ensure that the chosen employees represent all the departments of the two power plants.

\subsection{The procedure}

The questionnaire is handed to every employee in both plants personally. Everyone who received the questionnaire was informed about the aim of the questionnaire and the benefits of this work. The participants were told not to write their names. In addition, they were informed that the material is confidential and that nobody can see the individual results. The questionnaires were returned personally. Two hundred and ten questionnaires were distributed to all the employees. The return rate was $72.3 \%$.

For the interviews, 30 employees were selected using the stratified sampling method. Then, they were asked through emails if they would like to participate. The rate of responses to the interviews was $33.33 \%$ of the chosen employees. The chosen employees presented a good mixture from the two plants.

\subsection{Data analysis}

The data collected through the questionnaire was analyzed by Microsoft Office Excel. The raw data was used to calculate the mean value for each dimension for every participant, then the overall and group mean scores were calculated based on the individual results for each dimension (Cooper, 2001; Raja and Reghunath, 2010). Every two groups were examined for a significant difference using T-test with 95\% level of confidence and Probability value (P-value) less than 0.05 to consider the test as significant.

The internal consistency of each dimension was tested using Cronbach alpha coefficient; it is a coefficient of reliability, and the value of coefficient greater than 0.7 is considered ideal and reliable (George and Mallry, 2003; Gliem and Gliem, 2003; Pallant, 2007). Besides using the internal consistency, Interclass Correlation Coefficient (ICC) was used to determine the stability of the questionnaire.

\section{Results}

The overall mean score for both plants is 3.67 on a scale of 1 to 5, Table 1. The mean score for Plant A is greater than plant B in all seven dimensions. The mean scores for Plant A and Plant B are 3.93 and 3.49, respectively. The lowest mean score in all the seven dimensions is 2.83 obtained in dimension 4 for plant $\mathrm{B}$. 
Table 1: The Mean Scores and Standard Deviations of both Plant A and Plant B

\begin{tabular}{|c|c|c|c|c|c|c|c|c|}
\hline & DIM1 & DIM2 & DIM3 & DIM4 & DIM5 & DIM6 & DIM7 & Overall \\
\hline Mean value & 3.61 & 3.77 & 3.81 & 3.42 & 3.63 & 3.51 & 3.99 & 3.67 \\
\hline Mean value $\%$ & $65.3 \%$ & $69.3 \%$ & $70 \%$ & $60.5 \%$ & $65.8 \%$ & $62.8 \%$ & $74.8 \%$ & $66.8 \%$ \\
\hline Standard Deviation & 0.41 & 0.52 & 0.4 & 0.67 & 0.43 & 0.54 & 0.42 & 0.35 \\
\hline
\end{tabular}

The result of the T-test analysis shows that there is a significant difference in three dimensions, namely dimension 1 (Management safety priority and ability), dimension 4 (Workers' safety commitment) and dimension 6 (Peer safety communication learning and trust in safety ability), Table 2.

Table 2: The Variation in the Level of Safety Climate between Plant A and Plant B

\begin{tabular}{|c|c|c|c|c|c|c|c|c|c|}
\hline Site & $\mathrm{n}$ & DIM1 & DIM2 & DIM3 & DIM4 & DIM5 & DIM6 & DIM7 & Overall \\
\hline \multirow{2}{*}{ Plant A } & \multirow{2}{*}{$40 \%$} & 3.81 & 3.93 & 3.94 & 4.01 & 3.73 & 3.97 & 4.14 & 3.93 \\
\hline & & $70.25 \%$ & $73.25 \%$ & $73.5 \%$ & $75.25 \%$ & $68.25 \%$ & $74.25 \%$ & $78.5 \%$ & $73.25 \%$ \\
\hline \multirow{2}{*}{ Plant B } & \multirow{3}{*}{$60 \%$} & 3.53 & 3.71 & 3.73 & 2.86 & 3.62 & 3.13 & 3.88 & 3.49 \\
\hline & & $63.25 \%$ & $67.75 \%$ & $68.25 \%$ & $46.5 \%$ & $65.5 \%$ & $53.25 \%$ & $72 \%$ & $62.25 \%$ \\
\hline $\mathrm{P}$-value & & 0.035 & NS & NS & $2.14 \mathrm{E}-10$ & NS & $1.57 \mathrm{E}-8$ & NS & $5.9 \mathrm{E}-05$ \\
\hline
\end{tabular}

$\mathrm{DIM}=$ Dimension, $\mathrm{NS}=$ non-significant, $\mathrm{n}=$ percentage of participants, $\mathrm{P}$-value $=$ result of the T-Test

When compared between workers and managers/supervisors in all the seven dimensions in both plants, it is found that the overall is 3.67 and 4.13, respectively. Significance difference has been found in three dimensions, namely, dimension 1 (Management safety priority and ability), dimension 2 (Management safety empowerment) and dimension 5 (Workers' safety priority and risk non-acceptance), given in Table 3.

Table 3: The Variation in the Level of Safety Climate between the Workers and the Managers/Supervisors at Both Plants

\begin{tabular}{|c|c|c|c|c|c|c|c|c|c|}
\hline & $\mathrm{n}$ & DIM1 & DIM2 & DIM3 & DIM4 & DIM5 & DIM6 & DIM7 & Overall \\
\hline \multirow{2}{*}{ Workers } & \multirow{2}{*}{$83 \%$} & 3.61 & 3.77 & 3.81 & 3.42 & 3.63 & 3.51 & 3.99 & 3.67 \\
\hline & & $65.3 \%$ & $69.3 \%$ & $70 \%$ & $60.5 \%$ & $65.8 \%$ & $62.8 \%$ & $74.8 \%$ & $66.8 \%$ \\
\hline \multirow{3}{*}{$\begin{array}{l}\text { Managers/ Supervi- } \\
\text { sors } \\
\text { P-value }\end{array}$} & \multirow{3}{*}{$17 \%$} & 4.32 & 4.27 & 3.92 & 4.13 & 4.26 & 3.88 & 4.16 & 4.13 \\
\hline & & $83 \%$ & $81.75 \%$ & $73 \%$ & $78.25 \%$ & $81.5 \%$ & $72 \%$ & $79 \%$ & $78.25 \%$ \\
\hline & & 0.0002 & 0.048 & NS & NS & 0.002 & NS & NS & 0.0088 \\
\hline
\end{tabular}

$\mathrm{DIM}=$ Dimension, NS= non-significant, $\mathrm{n}=$ percentage of participants, $\mathrm{P}$-value $=$ result of the T-Test

By dividing the workers into two main categories (daytime and shift workers between the two plants), four groups were identified, namely, Plant A daytime workers, Plant A shift workers, Plant B daytime workers, and Plant B shift workers. The comparison between shift and daytime showed that daytime workers have a higher level of safety climate in both plants. Table 4 showed the results of safety climate analysis for each group.

Table 4: The Level of Safety Climate within Plant A and Plant B for Shift Workers and Daytime Workers

\begin{tabular}{|c|c|c|c|c|c|c|c|c|}
\hline Group & DIM1 & DIM2 & DIM3 & DIM4 & DIM5 & DIM6 & DIM7 & Overall \\
\hline \multirow{2}{*}{ Plant A Day time workers } & 4.06 & 4.23 & 4.06 & 4.04 & 3.89 & 3.98 & 4.29 & 4.07 \\
\hline & $76.5 \%$ & $80.75 \%$ & $76.5 \%$ & $76 \%$ & $72.25 \%$ & $74.5 \%$ & $82.25 \%$ & $76.75 \%$ \\
\hline \multirow{2}{*}{ Plant A Shift workers } & 3.64 & 3.71 & 3.85 & 3.98 & 3.61 & 3.95 & 4.04 & 3.82 \\
\hline & $66 \%$ & $67.75 \%$ & $71.25 \%$ & $74.5 \%$ & $65.25 \%$ & $73.75 \%$ & $76 \%$ & $70.5 \%$ \\
\hline \multirow{2}{*}{ Plant B Day time workers } & 3.84 & 3.91 & 3.81 & 2.83 & 3.75 & 3.39 & 4 & 3.64 \\
\hline & $71 \%$ & $72.75 \%$ & $70.25 \%$ & $45.75 \%$ & $68.75 \%$ & $59.75 \%$ & $75 \%$ & $66 \%$ \\
\hline \multirow{2}{*}{ Plant B Shift workers } & 3.28 & 3.54 & 3.67 & 2.89 & 3.51 & 2.92 & 3.79 & 3.37 \\
\hline & $57 \%$ & $63.5 \%$ & $66.75 \%$ & $47.25 \%$ & $62.75 \%$ & $48 \%$ & $69.75 \%$ & $59.25 \%$ \\
\hline
\end{tabular}

DIM= Dimension

When investigating the factors affecting the safety climate level, four factors were revealed clearly. 1) As the experience increased, the level of safety climate increased. 2) The workers with more workplace experience scored higher in safety climate level. 3) As the safety workshop numbers increased, it led to a higher safety climate level; 4) Also, the workers with more work experience in the plant itself scored higher levels of safety climate.

\section{Discussion}

The evaluation of the internal consistency of the questionnaire's seven dimensions showed positive results as the alpha coefficient values were between 0.55 and 0.81 , with an average of 0.73 . These results are considered accepted (George and Mallry, 2003). Also, the stability test showed positive results as it been found to be between 0.495 and 0.751 , which indicates acceptable stability for the questionnaire (Kines et al., 2011).

The minimum response rate for a survey to be acceptable depends on the size of the population (Basarab and Root, 1992). In the present work, a $72.3 \%$ response rate was achieved for a sample size of 210 . According to Basarab and Root, in 1992 the minimum acceptable response rate for a 200 -sample size is $66 \%$. The response rate for the interviews was $33.3 \%, 10$ out of 30 .

Overall safety climate level for both plants is considered above average. The overall mean score of all results is 3.67. The mean scores for most of the dimensions were higher than 3, which is considered a positive result (Bergh, 2011; Raja and Reghunath, 2010).

When investigating the two sites separately, a significant difference was found in dimension 1; (Management safety priority and ability), dimension 4; (Workers' safety commitment) and dimension 6; (Peer safety communication learning and trust in safety ability). This indicates there is a gap in the safety climate level between the two power plants A and B. The response to the interview questions can clarify some reasons behind this gap. The answers state that a difference may exist because the safety enhancement process started in plant A before plant B, alongside with the applying of standardized safety rules in plant A before plant B. Another reason, the locating of management in plant $\mathrm{A}$, gave it preference in adapting the safety rules and enhancing safety communication and reporting. In addition, according 
to Reason, 1997, the level of safety climate is affected by experiencing more accidents. When comparing the findings of Reason and the finding of the present work, the same conclusion is achieved. Referring to the mother company documents (2015), 65\% of the near misses occurred in plant B, while plant A has $35 \%$. According to present work results, plant B scored less on safety climate level.

Furthermore, when the safety climates between daytime workers and shift workers were compared, it was found that daytime workers have more safety climate level than shift workers. Previous studies have shown that working near the production line will affect the level of safety climate and make it lower than the safety climate level of the other workers who work far from the production site (Bergh et al., 2013; Collins and Gadd, 2002; Milczarek and Najmiec, 2004; Wu et al, 2007). This agrees with the present work results. The shift workers are working closer to the production site, which in this case includes gas turbines, steam turbines, energized equipment, and fuel gas-filled systems. Daytime workers work most of the time far of the production line, such as in workshops and stores.

Finally, comparing the safety climate levels between the workers and managers, the managers have higher levels of safety climate than the workers. One possible reason is that the safety issues discussed between the management and the safety department are not effectively communicated to the workers. This analysis is supported by the results of the interview answers.

\section{Conclusions}

The stability and reliability tests for the questionnaire showed acceptable results, which indicate that the designed questionnaire is a reliable tool for measuring the safety climate level in a power plant.

The results of studying the safety climate of both power plants A and B showed that the level of safety for both companies is above average 3.67 out of 5. The safety level of Plant A is 3.92, while for Plant B it is 3.49 . Therefore, plant B needs more attention in any safety improvement process.

Two sub-climates were detected within the company, daytime workers and shift workers. The sub-climate appears obviously more at Plant $B$ where the shift workers record a lower safety climate level.

The weaknesses are few. For the daytime workers of plant B, only one dimension showed weakness, which is dimension 4 (Workers' safety commitment). In addition, for the shift workers of plant B, two dimensions showed weaknesses, which are dimension 4 (Workers' safety commitment) and dimension 6 (Peer safety communication learning and trust in safety ability).

\section{References}

[1] Basarab D.J. and Root D.K., 1992. The Training Evaluation Process, Evaluation in Education and Human Services, 33, Chapter 2, Springer. https://doi.org/10.1007/978-94-011-2940-4_2.

[2] Bergh M., 2011. An evaluation of the safety climate at AkzoNobel Site Stenungsund, MSc Thesis, Chalmers University of Technology, Sweden.

[3] Bergh M., Shahriari M., Kines P., 2103. Occupational Safety Climate and Shift Work. Chemical Engineering Transactions 31: 403-408.

[4] CANSO, Safety Standing Committee, 2008. Safety Culture Definition \& Enhancement Process Model. Netherlands: The Civil Air Navigation Services Organization

[5] Collins A.M., Gadd S., 2002. Safety culture: A review of the literature. Sheffield: Health and Safety Laboratory, Human Factors Group, UK.

[6] Connelly L.M., 2008. Pilot studies. Medsurg Nursing, 17(6): 411-2. https://doi.org/10.12968/bjon.2008.17.7.29056.

[7] Cooper M.D., 2000. Towards a model of safety culture. Safety Science, 36: 111-136. https://doi.org/10.1016/S0925-7535(00)00035-7.

[8] Cooper M.D., 2001 Improving safety culture: A practical guide. Applied Behavioral Sciences Hull, UK.

[9] Flin R., Mearns K., O'Connor P., Bryden R., 2000. Measuring safety climate: Identifying the common features. Safety Science, 34: 177-192. https://doi.org/10.1016/S0925-7535(00)00012-6.

[10] George D., Mallery P., 2003. SPSS for Windows Step by Step: A simple guide and reference. 11 update. $4^{\text {th }}$ Ed. Boston: Allyn \& Bacon.

[11] Gliem J.A, Gliem R.R., 2003. Calculating, interpreting, and reporting Cronbach's Alpha Reliability Coefficient for Likert-type Scales. Columbus $(\mathrm{OH})$ : The Ohio State University, Midwest Research to Practice Conference in Adult, Continuing, and Community Education, USA.

[12] Guldenmund F.W., 2010. Understanding and Exploring Safety Culture. Uitgeverij Boxpress, Oisterwijk.

[13] Kines P., Lappalainen J., Mikkelsen K.L., Olsen E., Pousette A., Tharaldsen J., Tómasson K., Törner M., 2011. Nordic Safety Climate Questionnaire (NOSACQ-50): A new tool for diagnosing occupational safety climate. International Journal of Industrial Ergonomics, 41: 634-646. https://doi.org/10.1016/j.ergon.2011.08.004.

[14] Kinik Y., 2010. Improving Safety Culture: A study performed at KLM Engineering and Maintenance. France.

[15] Milczarek M., Najmiec A., 2004. The Relationship between Workers' Safety Culture and Accidents, Near Accidents and Health Problems. International Journal of Occupational Safety and Ergonomics, 10 (1) 25-33. https://doi.org/10.1080/10803548.2004.11076592.

[16] Pallant J., 2007. SPSS Survival Manual. $3^{\text {rd }}$ Ed. Berkshire: McGraw Hill; UK.

[17] Parsian N., Dunning D., 2009. Developing and Validating a Questionnaire to Measure Spirituality: A Psychometric Process. Global Journal of Health Science, 1(1): 2-11. https://doi.org/10.5539/gihs.v1n1p2.

[18] Raja S.V., Reghunath K.P., 2010. Empirical Analysis of Construction Safety Climate - A Study. International Journal of Engineering Science and Technology, 2(6): 1699-1707.

[19] Reason J., 1997. Managing the Risks of Organizational Accidents. Aldershot: Ashgate Publishing Limited.

[20] Schein E.H., 2010. Organizational Culture and Leadership. San Francisco: Jossey-Bass.

[21] Wu T.C.; Liu C.W.; Lu M.C., 2007. Safety climate in university and college laboratories: Impact of organizational and individual factors. Journal of Safety Research, 91-102. https://doi.org/10.1016/j.jsr.2007.01.003. 